\title{
Imaginarios urbanos y segregación socioespacial. Un estudio de caso sobre Quito*
}

\begin{tabular}{|l|l|l|}
\hline \multicolumn{2}{|l|}{ Fecha de recepción: 24 de agosto de 2015 Fecha de aceptación: 18 de septiembre de 2015 Disponible en línea: 30 de octubre de 2015} \\
Alfredo Santillán Cornejo \\
\hline Doctorando en Estudios Sociales & Docente e investigador del Departamento de Antropología, Historia y Humanidades \\
\hline Facultad Latinoamericana de Ciencias Sociales (Flacso), sede Ecuador & asantillan@flacso.edu.ec \\
\hline
\end{tabular}

Resumen El artículo expone los resultados de la aplicación del marco conceptual y metodológico de imaginarios urbanos de un estudio de caso sobre la ciudad de Quito como herramienta útil, con el fin de comprender la forma como se representa la segregación socioespacial que ha caracterizado históricamente a la capital ecuatoriana. Los hallazgos obtenidos apuntan a que la adscripción espacial constituye un elemento determinante de los imaginarios que dan sentido a las fronteras intraurbanas y, de esta manera, las producciones imaginarias permiten reconstruir, en el plano simbólico, las tensiones que conllevan las desigualdades sociales y espaciales.

encuesta; imaginarios urbanos; Quito; segregación urbana

\footnotetext{
Artículo de investigación científica y tecnológica. Presenta los datos obtenidos en el proyecto de investigación "Espacio urbano y subjetividades. Disputas simbólicas en torno a los imaginarios sobre el sur de Quito", desarrollado en el año 2013 y financiado por el Fondo de Desarrollo Académico de Flacso Ecuador.
}

Cómo citar este artículo: Santillán Cornejo, A. (2015). Imaginarios urbanos y segregación socioespacial. Un estudio de caso sobre Quito. Cuadernos de Vivienda y Urbanismo, 8(16), 246-263. http://dx.doi.org/10.11144/Javeriana.cvu8-16.iuss 


\section{Urban Imaginaries and Sociospatial Segregation. A Case Study on Quito}

Abstract The article presents the results of applying the conceptual and methodological framework of urban imaginaries of a case study on the city of Quito as a useful tool in order to understand how the sociospatial segregation that has historically characterized the capital of Ecuador is represented. The findings suggest that the spatial assignment constitutes a key element for the imaginaries that give meaning to the intra-urban boundaries and thus the imaginary productions allow us to reconstruct, on a symbolic level, the tensions that social and spatial inequalities entail.

Keywords survey; urban imaginaries; Quito; urban segregation

\section{Imaginários urbanos e segregação socio-espacial. um estudo de caso sobre Quito}

Resumo Seguindo um estudo de caso sobre a cidade de Quito, o artigo expóe os resultados da aplicação de um marco conceitual e metodológico dos imaginários urbanos como ferramenta útil no proposito de compreender a maneira de representar a segregação sócio-espacial que caracteriza historicamente a capital equatoriana. As constataçóes obtidas apontam que a vinculação espacial é um elemento determinante dos imaginários que dão sentido ás fronteiras intraurbanas e dessa forma as produçóes imaginárias permitem reconstruir, no plano simbólico, as tensóes vinculadas ás desigualdades sociais e espaciais. 


\section{Introducción}

“CCómo hablar de la ciudad-cosa (casa) o de la 'cosa en sí misma', de la cual, entre otros ilustres profesionales, se ocupan los arquitectos, cuando sabemos que la percepción es parte de esa cosa-casa?" (Silva, 2004, p. 84). Armando Silva plantea este interrogante dentro de la formulación teórica de "imaginarios urbanos" con el fin de problematizar la referencia permanente a la ciudad como estructura física que deja de lado su representación por parte de los ciudadanos; lo que él llama la "ciudad imaginada". Esta pregunta sintetiza una mirada a los fenómenos urbanos que surge de reconocer que toda "realidad" es una representación, pues no existe por fuera de las operaciones cognitivas y discursivas que la hacen existir y, por tanto, entra en tensión con las visiones, muy comunes en la discusión sobre las problemáticas urbanas, que reducen la ciudad a su materialidad arquitectónica.

Más allá de la provocación del autor a debatir acerca de la experiencia subjetiva de la ciudad, este cuestionamiento es concurrente con un amplio movimiento académico enfocado en construir un diálogo interdisciplinario o transdisciplinario, que permite conjugar los componentes materiales y simbólicos que constituyen la espacialidad humana. Algunos autores hablan de este movimiento como un "giro espacial en las Ciencias Sociales" o como un "giro cultural" en la disciplina particular de la Geografía (Giménez, 2001; Lindón, 2012; Peña, 2011). El aporte a este diálogo de la teorización de Silva es la atención que pone en elementos subjetivos como las emociones, la imaginación, la evocación, etc. en la constitución de lo urbano.
Este marco conceptual no se limita a identificar la representación de la ciudad como el objeto de estudio en sí, sino que la entiende como un vehículo para la expresión de construcciones intersubjetivas más profundas, que el autor denomina "imaginarios urbanos". El concepto no es una formulación exclusiva del pensamiento de este autor, pues tiene un campo semántico variable en distintos autores, de acuerdo con las concepciones que se tenga de los dos términos que están en juego: el imaginario y lo urbano. De ahí que su uso suele ser impreciso y requiere un trabajo de esclarecimiento.

Varios autores señalan que la introducción del concepto de "imaginario" en trabajos orientados a comprender prácticas relacionadas con la ciudad ha permitido volver la mirada al campo cultural como componente vital de la vida urbana, pero su mayor limitante es la falta de sistematicidad teórica que delimite los fenómenos que pueden ser comprendidos con esta categoría y de sistematicidad metodológica para dar cuenta de ellos. Así, con base en esta acepción, se han estudiado las prácticas cotidianas, los usos y las apropiaciones de los espacios físicos, las representaciones de la ciudad y sus partes (por ejemplo, los centros históricos) o algunas interconexiones directas entre usos y representaciones como el miedo al "otro" y la consiguiente fortificación urbana (Hiernaux, 2007; Girola, 2012).

La acepción de Silva (2007) es un constructo que se nutre de insumos teóricos situados en el Psicoanálisis, la Filosofía y la Semiótica. Uno de sus mayores logros es haber generado una agenda de 
investigación empírica a partir de la cual el trabajo teórico se nutre de los datos obtenidos, lo que no solo permite "poner a prueba" la teoría, sino encausar el mismo proceso de teorización. El autor sintetiza este trabajo teórico en la siguiente definición de "imaginario urbano":

[...] proceso psíquico perceptivo cuando lo entendemos motivado por el deseo y cuando lo que atendemos no es su representación ni su descarga satisfactoria sino una forma de aprehender el mundo [...]. Si queremos comprender los modos como aprehendemos el mundo desde una posición de sujetos deseantes, estamos proyectando los imaginarios, lo que incluye tanto el proceso lógico como el hermenéutico y analítico. Pero esa posición deseante ha de ser social, pues los imaginarios han de agregar otra premisa que ya señalamos: son colectivos - y por ello públicos — y se estudian desde los modos sociales de conocer (p. 91).

Una de las características fundamentales de esta formulación es que los imaginarios "hacen aparecer" las representaciones y establecen también los modos de percibir la ciudad; por ende, configuran un recurso cognitivo que no se queda en la mente, sino que encarna en la praxis que constituye lo urbano. Además, esta concepción avanza en identificar el trasfondo del imaginario, porque no lo reduce a la faceta inventiva de la fabulación - aunque no desmerece la creatividad con la que opera - y vincula la acción de imaginar con el sustrato de lo social, que es el deseo. Se imagina por la necesidad de dar respuesta a algo que está irresuelto y, por ello, es una actividad indispensable para la misma existencia.

Esta explicación de la base conceptual de la noción de imaginario lleva a problematizar la representación de los espacios urbanos de otra manera. Si partimos de que el imaginario pone de manifiesto las situaciones irresueltas de trascendental importancia en el juego social, es posible preguntarnos ¿por qué se privilegia la representación de ciertos lugares en vez de otros? y ¿por qué se los representa de cierta manera y no de otras posibles? Estas cuestiones apuntan a señalar que estas representaciones no son casuales ni inocuas; por el contrario, en ellas se muestra la significación de los lugares y entran en disputa sus condiciones de existencia social.

Dentro de la crítica a los estudios sobre imaginarios urbanos (no solo los impulsados por Silva) se destaca como limitación fundamental su desvinculación de las lógicas estructurales que marcan, en gran medida, la pauta de los procesos urbanos. Gorelik (2002), por ejemplo, cuestiona la desconexión de estos trabajos respecto a las lógicas económicas y políticas excluyentes que caracterizan, sobre todo, a las ciudades latinoamericanas. Los estudios sobre imaginarios darían cuenta de una cotidianidad urbana de manera ingenua, cuando no "despolitizada": mientras los ciudadanos experimentan la vida urbana a partir de las fabulaciones, se despliega la lógica mercantil de lo que varios autores denominan la "ciudad neoliberal", caracterizada por la importancia del espacio urbano en la acumulación capitalista (Hiernaux, 2007; Gorelik, 2002; De Mattos, 2010).

En esta discusión, el presente artículo plantea la posibilidad de utilizar la propuesta teórica y metodológica de Silva para el estudio de imaginarios urbanos, con miras a comprender uno de los fenómenos históricos característicos de las ciudades latinoamericanas: la segregación socioespacial. Para esto, se toma el caso de la ciudad de Quito, que cuenta con el rasgo particular de la persistencia de una representación dual de la ciudad, segmentada en un norte asociado con la modernización permanente, el estatus e incluso los privilegios sociales y un sur ligado a lo popular, la pobreza y, en general, la subalternidad. Los datos utilizados constituyen una síntesis de la información de campo obtenida mediante la aplicación de una encuesta realizada durante el año 2013. 


\section{Breve genealogía del imaginario de la segregación norte-sur}

Existe una considerable literatura respecto a la ciudad de Quito, centrada en exponer que la frontera intraurbana que caracteriza a la ciudad es la oposición entre el norte y el sur (Aguirre, Carrión y Kingman, 2005; Carrión, 1987; Achig, 1983; Unda, 1992; Naranjo, 1999). Dicha bibliografía muestra la formación histórica de esta división tanto en el plano material - por medio de las políticas de planificación urbana de mediados del siglo XX - como en el plano simbólico -mediante la ideología de separación de clases sociales que inspiró tales decisiones urbanísticas-. Más adelante, se argumenta la relativa desfiguración de la frontera a partir de la década del setenta, al menos en el plano material, cuando el crecimiento de la ciudad alcanzó lo que Carrión (1987) definió como "etapa metropolitana", en la cual se conforma un cinturón de barrios precarios que bordea el sur y el norte de la ciudad.

En resumidas cuentas, la historia de la segregación socioespacial inició a principios del siglo $\mathrm{XX}$, con el desplazamiento de las élites hacia el sector norte en relación con el casco histórico-colonial, mientras hacia el sur se planificó el desarrollo de los "barrios obreros", en función de la cercanía con la naciente zona industrial y el centro quedó como sector de residencia para clases medias vinculadas al aparato administrativo ubicado en esa zona. Esta manera de instituir las modernas divisiones de clase en el espacio urbano estuvo impulsada, en primera instancia, por las élites y luego, legitimada por el municipio de Quito, según lo documentan varias investigaciones (Achig, 1983; Carrión, 1987). Estos trabajos resaltan que dicha institución tuvo la tensión de responder, desde la Administración pública local, a los intereses privados de las clases dominantes $y$, al mismo tiempo, la necesidad de "racionalizar" el crecimiento de la urbe, al regular las lógicas especulativas impuestas por las mismas élites.

Otros trabajos históricos sobre la ciudad, centrados en el estudio de los conflictos de clase y raza como elementos constitutivos de las sociedades andinas, muestran que este desplazamiento obedecía también al principio de búsqueda de homogeneidad social legado de la época colonial, cuando la apuesta por habitar "barrios separados" permitía garantizar la reproducción de grupo amenazada por la posible "mezcla social" (Kingman, 2006; Ibarra, 1998). Esta visión permite pensar la relación entre el orden material y el orden simbólico de la ciudad constituido no solo por los intereses económicos, sino también por los sistemas de valores y los conflictos culturales.

La complejidad de este proceso ha sido bastante estudiada en su conformación histórica, en la que es posible plantear la correspondencia entre el orden material segregado y el imaginario dual de la ciudad. Desde la década del ochenta se puede identificar una paradoja muy interesante: en tanto los trabajos sobre la conformación material de la ciudad señalan que a partir de la década del setenta se desdibuja la dicotomía entre el norte como reducto de los grupos de mayores recursos y el sur como el espacio propio de las clases populares (Carrión, 1987; Unda, 1992); los análisis de carácter cultural muestran la vigencia de la representación dual hasta la actualidad, como narrativa que "espacializa" las diferencias sociales en la ciudad (Aguirre, Carrión, y Kingman, 2005; Naranjo, 1999; Ibarra, 1998).

Si bien el sur se ha consolidado paulatinamente mediante la implementación de infraestructuras de servicios importantes y desde hace varias décadas es habitado por clases medias con un importante poder adquisitivo, es representado como "desfavorecido" frente al norte. A su vez, 
pese a que en el norte están ubicadas grandes barriadas precarias, se mantiene vigente su significación como "pudiente". Esta dislocación entre orden material y orden simbólico es característica de las construcciones imaginarias, como lo propone Silva (2013), en tanto la ficción se impone a la realidad fáctica. Pese a la naturalización de esta dicotomía en el sentido común, la frontera resulta imprecisa, como señalan los autores del estudio Quito imaginado: "[...] nadie sabe a ciencia cierta dónde comienza el norte y dónde el sur. Se trata de fronteras móviles. Ni el norte ni el sur ocupan espacios fijos" (Aguirre, Carrión, y Kingman, 2005, p. 48).

Para abordar el tema, es necesario precisar el vocabulario y, en tal propósito, se propone diferenciar entre los términos Norte y Sur como coordenadas de ubicación geográficas en la ciudad de las expresiones norte y sur como lugares figurativos que condensan una poderosa carga histórica que localiza la posesión o no de distintos privilegios sociales. Debido a la carga valorativa asociada con estos términos, su utilización sugiere características sociales por medio de la localización; no obstante, aunque en su uso común se juega indistintamente entre su significación geográfica y su connotación social, el norte y el sur son asumidos a priori como diferentes, pero existe una gran imprecisión sobre en qué radica esa distinción.

La puesta en marcha de la investigación pretendió "dar forma" a un escenario disperso de producción de sentidos sobre la oposición entre ambas zonas, con énfasis en el análisis entre las diferencias y similitudes de las percepciones en función de la ubicación del lugar de residencia de las personas encuestadas, a fin de relacionar la localización del informante con las percepciones y valoraciones sobre cada zona.

\section{Algunos aspectos metodológicos}

En principio, el diseño de la encuesta siguió la propuesta metodológica de "imaginarios urbanos" (Silva, 2004, p. 28), la cual consiste en una "encuesta-entrevista" con un alto porcentaje de preguntas abiertas que faciliten la fluidez del diálogo en la captura de información. Como parte de esta técnica, la "asociación libre" en la elaboración de respuestas cumple un papel fundamental, en tanto permite captar las significaciones inmediatas de las personas encuestadas, porque en ellas afloran los estereotipos, las creencias inconscientes y las fabulaciones colectivas que resultan reveladoras de las construcciones imaginarias. Este modelo está pensado para muestras reducidas -máximo trescientas encuestas - para identificar pequeñas tendencias de respuestas comunes como señal de percepciones que puedan calificarse como colectivas (Silva, 2004).

Para lograr los objetivos del estudio, se trabajó con un esquema de encuesta con algunas variantes. En primer lugar, se adecuó el formulario con un alto margen de campos no estructurados, con preguntas abiertas, en lugar de la entrevista propuesta en la metodología original, pues se trabajó con una muestra de 930 encuestas, con el fin de garantizar la representatividad de las variables. En segundo lugar, se introdujo la variable analítica "sector", definida como la localización del lugar de residencia del encuestado en las zonas Norte, Centro y Sur de la ciudad $^{1}$. El modelo original de la encuesta propuesta por Silva contempla las variables de nivel socioeconómico (en adelante, NSE), género y edad, entendidas como cualidades que producen "puntos de vista" diferenciados de apreciación sobre la ciudad (Silva, 2004). En este 
escenario, la introducción de la variable sector buscó establecer si la ubicación del encuestado en un lugar específico dentro de un orden material o simbólico segregado constituye un punto de vista particular desde donde se construye también la mirada sobre la misma segregación.

La muestra se diseñó para garantizar representatividad de los sectores Sur, Centro y Norte de Quito, con un error muestral de $\pm 3,3$ para la muestra total y de $\pm 5,6$ a $\pm 5,8$ para la muestra de cada sector. La selección de las zonas específicas en las que se aplicaron las encuestas se hizo con un método de selección aleatoria simple, mediante la acumulación de frecuencias de población y la definición de un período a partir de un número aleatorio. El valor del período resultó de 8,479 para la población de la ciudad mayor de 15 años, de acuerdo con el censo nacional de 2010. El número de encuestas aplicadas para cada sector, NSE y edad no corresponde al peso real que tiene cada segmento en el universo. Para lograr la paridad entre muestra y universo en el procesamiento, se introdujeron índices de ponderación. El Anexo 1 presenta los detalles de la muestra aplicada.

\section{Resultados}

A continuación se presentan algunos datos ilustrativos del debate teórico, agrupados en tres secciones: la primera evidencia la vigencia de la percepción diferenciada entre las zonas norte y sur, en la cual los atributos característicos de cada una expresan una visión jerárquica. La segunda identifica asociaciones de cada zona con palabras que las caractericen, lo que produce metáforas que refuerzan la distinción. La tercera sección presenta datos de la caracterización de la población que reside en cada zona en donde las diferenciaciones espaciales se expresan también como atributos diferentes de sus habitantes.

\section{Norte-sur. La diferencia como jerarquía}

En el diseño del cuestionario se vigiló que las preguntas permitieran captar las percepciones sobre cada zona tratando de reducir al mínimo la inducción de valoraciones particulares, al prestablecer la demarcación entre Norte-Centro-Sur. Para ello, resultó útil el uso mayoritario de preguntas abiertas y metafóricas; sin embargo, el cuestionario también incluyó preguntas cerradas explícitas, cuyas respuestas arrojaron tendencias muy marcadas. Lo más significativo del uso de este recurso se obtuvo en la aplicación de un conjunto de cinco preguntas cerradas que se complementan entre sí y en las que se proponen diversas afirmaciones, frente a las cuales las personas encuestadas expresaron su acuerdo o desacuerdo. Tres de las cinco preguntas plantean un grado de diferenciación entre ambas zonas y las definen como "mundos distintos", "muy parecidos" y "no existen diferencias"; las dos afirmaciones restantes anteponen una zona sobre la otra, a partir de la idea de contar con "mejores lugares"3. Los resultados obtenidos se ven en el Gráfico 1.

El dato más destacado, en términos de tendencia de respuesta, es el mayoritario desacuerdo con la idea de que el "norte y el sur son muy parecidos". Esto se evidencia en el alto porcentaje de desacuerdo con la frase de que "no existen diferencias" entra ambas zonas y en el acuerdo con la idea de que "el norte y el sur son mundos distintos" (Gráfico 1). Por su parte, las respuestas acerca de la valoración jerarquizada de ambas zonas también se corresponden, pues la mayor frecuencia se presenta en el desacuerdo con la idea de que "el sur tiene mejores lugares que el norte", reforzada por el acuerdo con que "el norte tiene mejores lugares que el sur” (Gráfico 1).

Esta expresión fue recogida en varias conversaciones previas al levantamiento de la encuesta con habitantes tanto del norte como del sur, en la que definían el paso de una zona a otra como "atravesar mundos distintos". 
Gráfico 1.

Acuerdo y desacuerdo con las afirmaciones propuestas

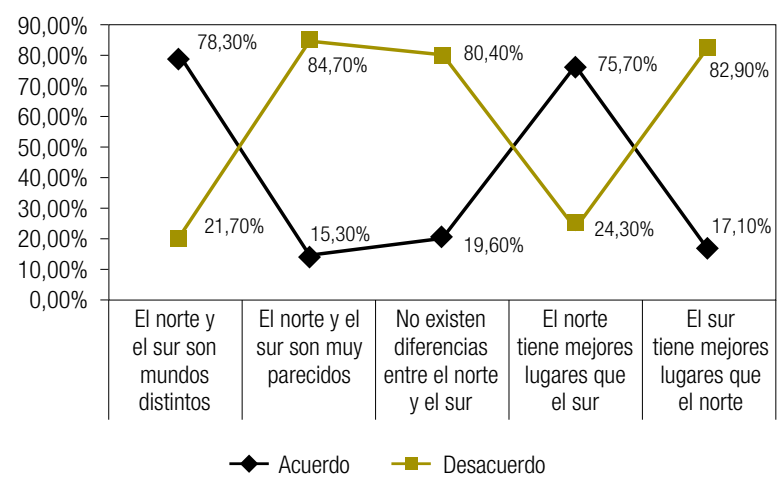

Fuente: elaboración propia

La desagregación de los datos por sector arroja información relevante sobre cómo se componen las valoraciones de mayor consenso que muestran algunas variaciones significativas respecto a las tendencias generales. A continuación se presenta la desagregación de las dos afirmaciones que condensan los acuerdos más sobresalientes sobre la diferencia norte-sur antes mencionados (Gráficos 2 y 3). En términos generales, existe cierta homogeneidad de las distintas variables, pues las diferencias en la mayoría de los casos operan dentro de rangos pequeños. No obstante, vale la pena señalar la importancia de la variable sector que resulta decisiva en la generación de puntos de vista diferenciados.

\section{Gráfico 2.}

Desagregación por sector, NSE, género y edad de acuerdo con la afirmación: "El norte y el sur son muy parecidos"

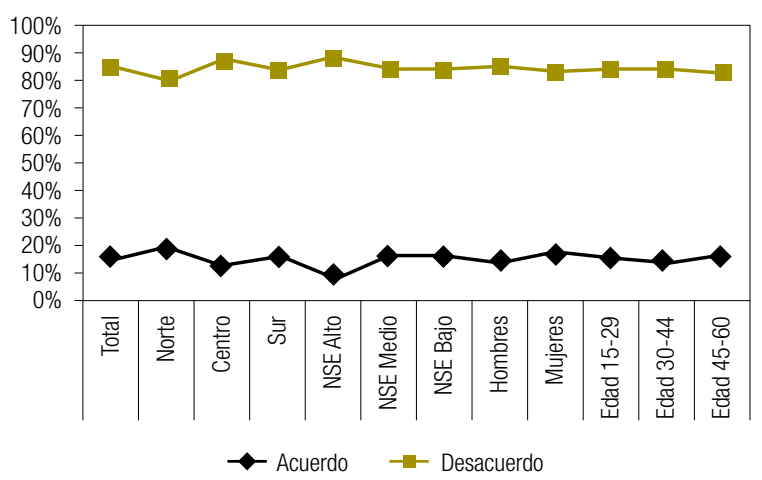

Fuente: elaboración propia
En el primer caso, en la posición frente a la afirmación "el norte y el sur son muy parecidos", las variables de género y edad son bastante homogéneas, mientras las variables de NSE y sector muestran las mayores variaciones. Así, son las personas de NSE alto quienes, en su mayoría, desaprueban la idea de la semejanza entre ambas zonas respecto al resto de estratificaciones por NSE; algo similar sucede con la variable sector, pues la población del Sur, en comparación con la del Centro y el Norte, es más enfática en rechazar la idea de similitud entre ambas zonas (Gráfico 2). En el segundo caso, si bien hay un marcado consenso en rebatir la idea de que el sur tenga "mejores lugares" que el norte - lo que confirma la valoración jerárquica del norte-, en la desagregación de datos aparece una mayor heterogeneidad en todas las variables. Las mayores frecuencias de acuerdo con esta frase se ubican en el sector Sur, en el NSE medio y entre las mujeres y la población de mayor edad (Gráfico 3).

A partir de los datos obtenidos de las respuestas a las preguntas que indagan por la valoración de ambas zonas, es posible establecer que el norte y el sur como lugares figurativos no solo son representados como diferentes, sino como espacios jerarquizados. Esta significación hegemónica que acentúa la diferencia entre ambas zonas sobre las semejanzas confirma su institución como

Gráfico 3.

Desagregación por sector, NSE, género y edad de acuerdo con la afirmación: "El sur tiene mejores lugares que el norte"

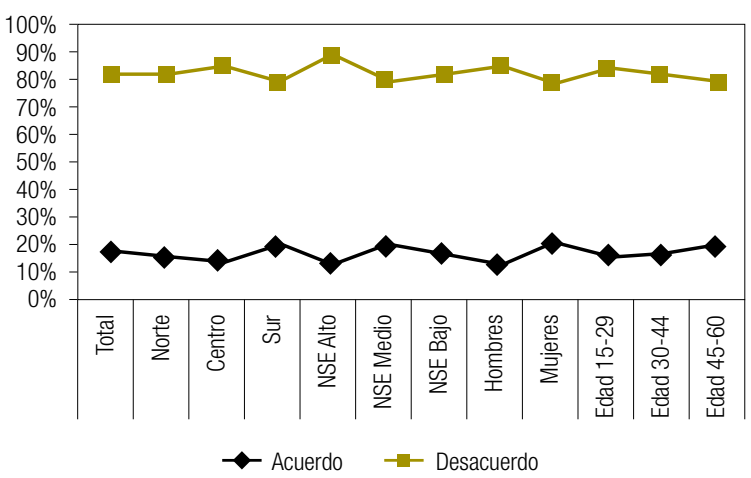

Fuente: elaboración propia 
imaginario, en tanto la creencia en la dualidad como construcción subjetiva compartida sobrepasa la evidencia empírica de una ciudad dual. Los datos de los siguientes apartados permiten profundizar en esta interpretación.

\section{Asociación libre y representación de la diferencia}

Otro bloque del cuestionario aplicado buscó revisar las representaciones de ambas zonas de manera indirecta, es decir, por medio de asociaciones libres con palabras o imágenes que definan a cada zona de la ciudad. Una característica importante de las respuestas obtenidas es que, pese a que las preguntas fueron abiertas, las respuestas son coincidentes en porcentajes importantes, lo que indica marcos de significación comunes. A continuación se presenta la información obtenida en la caracterización mediante palabras (Cuadro 1).

Cuadro 1.

Palabra o imagen que caracterice al norte

\section{Palabras agrupadas}

Porcentaje

\begin{tabular}{|cc|}
\hline $\begin{array}{c}\text { Bonito / lindo / hermoso / bello / atractivo / simpático } \\
\text { Tranquilo / tranquilidad / calmado / pacífico }\end{array}$ & $14,80 \%$ \\
\hline $\begin{array}{c}\text { De la alta } \\
\text { / aniñados }{ }^{5} \text { / pelucón }\end{array}$ & $12,50 \%$ \\
\hline Grandencia / adinerado & $10,60 \%$ \\
\hline Aseado / limpio / pulcro & \\
\hline Comercial / muy comercial & $3,80 \%$ \\
\hline Bueno & $3,70 \%$ \\
\hline No sabe & $2,90 \%$ \\
\hline Agradable / acogedor & $2,60 \%$ \\
\hline Seguro / seguridad & $2,40 \%$ \\
\hline Elegante / lujoso & $2,20 \%$ \\
\hline Moderno / medio moderno / modernización & $2,10 \%$ \\
\hline Otros & $2,00 \%$ \\
\hline
\end{tabular}

Fuente: elaboración propia
Las frecuencias de estas apreciaciones muestran en primer lugar que, si se omiten, por un lado, las respuestas más dispersas agrupadas en la categoría de "otros" y, por otro, la opción "no sabe", las palabras utilizadas comparten una valoración positiva que exalta en el norte distintos atributos como "bonito", "tranquilo", "espacioso", "limpio", "agradable”, aunque resulta ambigua una de las mayores percepciones, como la asociación con la posición económica en expresiones como "de la alta" (y sus similares: "aniñado", "pelucón”, “opulencia”, "adinerado”). Si bien reconoce el valor diferencial de la posesión económica, contiene una connotación crítica de esta condición, al sancionar la desigualdad implícita en la posesión de privilegios sociales. En los datos de las variables desagregadas aparecen diferencias bastante significativas. Para fines expositivos se presentan en el Gráfico 4 los datos de las tres principales respuestas.

\section{Gráfico 4}

Desagregación por sector, NSE, género y edad: palabra 0 imagen que caracterice al norte

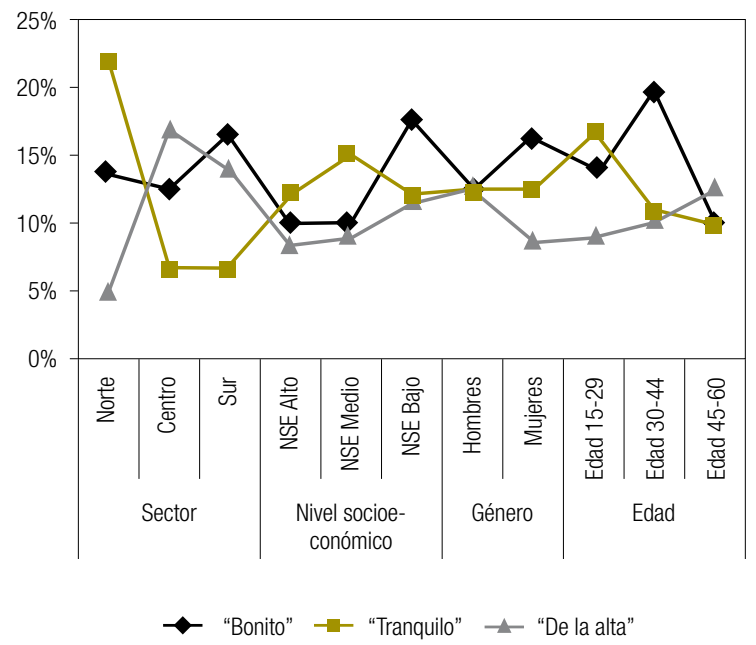

Fuente: elaboración propia 
En relación con la palabra que caracteriza al "norte", hay diferencias marcadas en todas las variables, pero el sector presenta las mayores divergencias en comparación con las otras (Gráfico 4). El uso del calificativo "bonito" presenta muy pocas variaciones entre las tres zonas y constituye el mayor acuerdo en la descripción del "norte". Los mayores desacuerdos están en el uso de los calificativos "tranquilo" y "de la alta"; es solo para la población que reside en el Norte la cualidad que mejor lo define es la de "tranquilo" y la menos usada es "de la alta". En contraste, esta última expresión está entre las más usadas por la población del Centro y del Sur y "tranquilo" es de las menos representativas. En definitiva, se puede decir que la apreciación de la población del Norte sobre su zona es distinta a la de los otros sectores de la ciudad, en tanto minimiza el rasgo que contiene la connotación crítica a los privilegios sociales.

En cuanto a la palabra o imagen que caracteriza al sur aparecen contrastes muy importantes (Cuadro 2).

Cuadro 2.

Palabra o imagen que caracterice al sur

\begin{tabular}{cc} 
Palabras agrupadas & Porcentaje \\
\hline Bonito / lindo / hermoso / bello / atractivo / simpático & $9,60 \%$ \\
\hline Peligroso / delincuencia / choros ${ }^{7}$ & $7,20 \%$ \\
\hline Alegre / divertido / diversión & $6,50 \%$ \\
\hline Tranquilo / tranquilidad / calmado / pacífico & $4,70 \%$ \\
\hline No sabe & $4,30 \%$ \\
\hline Comercial / muy comercial & $3,70 \%$ \\
\hline Pobreza / pobres / más pobre & $3,40 \%$ \\
\hline Inseguro / intranquilo / nada seguro & $3,30 \%$ \\
\hline Popular & $2,90 \%$ \\
\hline Normal / común y corriente & $2,10 \%$ \\
\hline Grande / espacioso / holgado / amplio / amplitud & $2,00 \%$ \\
\hline En desarrollo / avance / evolución & $1,70 \%$ \\
\hline Otros & $48,40 \%$ \\
\hline
\end{tabular}

Fuente: elaboración propia

"Choros" significa ladrones en el argot local.
Las respuestas obtenidas a esta pregunta marcan algunas diferencias sustanciales frente a las obtenidas acerca del norte. En primer lugar, la heterogeneidad de respuestas es significativamente mayor, tanto así, que la frecuencia de las opciones que alcanzan porcentajes menores a $1,5 \%$ es casi la mitad del total de respuestas ( $48,4 \%$ frente a 38,2\% que representa en el caso del norte). En segundo lugar, las respuestas que alcanzan los mayores porcentajes no llegan a los grados de coincidencia que se pueden ver en las respuestas obtenidas en el caso del "norte"; a pesar de que en ambos casos la frecuencia más alta corresponde al calificativo de "bonito", en el caso del sur corresponde a un 9,6\%, mientras en el caso del norte es de $14,8 \%$, diferencia bastante alta, si se considera que se trata de una pregunta abierta.

En tercer lugar, la diferencia más importante es que los calificativos del sur oscilan entre valoraciones positivas como "bonito", "alegre" y "tranquilo" y negativas como "peligroso", "pobreza" e "inseguro", lo que indica que no existe un acuerdo tan marcado sobre su valoración social, como lo es para el caso del norte y su asociación con el bienestar (Cuadro 2). Esta heterogeneidad permite pensar que la significación del sur es un campo en disputa, en el que están en juego distintas formas de dar sentido a la diferenciación social mediante la tensión entre los atributos positivos y negativos. La desagregación de datos presentada en el Gráfico 5 permite profundizar en estas diferencias.

En la desagregación de la palabra que caracteriza al "sur', al igual que en el caso del "norte', si bien hay una gran heterogeneidad en todas las variables, la variable sector presenta las diferencias de respuesta más radicales. El adjetivo "bonito", en cuanto a sectores, se localiza sobre todo en el Sur, con una gran diferencia frente a las otras zonas; en contraste, el adjetivo de "peligroso" se localiza fundamentalmente en la población del 
Norte y resulta muy bajo en las otras zonas. Así, la población del Sur es la que construye, en su mayoría, una representación positiva de su zona con el calificativo de bonito y, a su vez, la mayor valoración negativa con el calificativo "peligroso" se concentra en la población del Norte. En el plano de la imagen negativa de cada zona se produce una diferencia importante: mientras la visión negativa del "sur" se presenta en proporción mayoritaria en la población del Norte, la visión negativa del "norte" resulta compartida entre la población del Sur y del Centro.

Gráfico 5.

Palabra 0 imagen que caracterice al sur según sector, NSE, género y edad muy importante es que, pese a la gran cantidad de posibles contestaciones, las palabras expresadas por cada encuestado resultan bastante coincidentes, de forma que un alto porcentaje de ellas corresponde a un pequeño grupo de cerca de veinte términos (considerados sus sinónimos o conceptos directamente asociados) tanto en el caso del norte como del sur (Cuadro 3).

Tras un análisis hermenéutico sobre las palabras empleadas para describir las características de la población de cada zona, pueden identificarse dos grandes ejes que articulan los calificativos que son, por un lado, la condición económica y, por otro, cualidades morales. En ambos casos, los términos se polarizan entre valoraciones positivas y negativas; así, la condición económica positiva puede denominarse "posesión económica" y la negativa, "carencia económica". Asimismo, las cualidades morales positivas pueden denominarse "virtudes morales" y las negativas "defectos morales". Desde esta lógica de clasificación, se pueden hacer comparaciones entre los conceptos que aparecen en ambas zonas frente a los términos que aparecen únicamente para alguna de las dos (Cuadro 4).

En esta comparación en cuanto al campo de la condición económica no existen términos comunes para ambas zonas, sino que se polarizan, con énfasis en la posesión económica como cualidad de la gente del norte frente a la carencia económica de la población del sur. Esto implica una condición muy importante de las producciones imaginarias en las cuales los atributos asignados al lugar pueden desplazarse como caracterización de las personas que lo habitan y viceversa.

Respecto al campo de las cualidades morales, las palabras que aparecen en ambas zonas corresponden, en exclusiva, al campo de las virtudes morales, pues no se identifican defectos morales similares en ambas zonas. Sin embargo, la 
Cuadro 3.

Términos usados en la caracterización de la población del norte y del sur

\begin{tabular}{|c|c|c|c|c|c|}
\hline \multicolumn{3}{|c|}{ Términos para el Norte } & \multicolumn{3}{|c|}{ Términos para el Sur } \\
\hline Principal & Sinónimos & $\%$ & Principal & Sinónimos & $\%$ \\
\hline Pelucones & Aniñados & $20.7 \%$ & Sociables & Amigables, abiertos, tratables & $22.8 \%$ \\
\hline Amables & Corteses, atentos, gentiles & $18.8 \%$ & Amables & Corteses, atentos, gentiles & $17.4 \%$ \\
\hline No sabe & & $12.4 \%$ & No sabe & & $15.1 \%$ \\
\hline Educados & Profesionales & $12.2 \%$ & Divertidos & Contentos, alegres, joviales & $9.8 \%$ \\
\hline Creídos & Alzados, engreídos & $11.7 \%$ & Humildes & & $9.1 \%$ \\
\hline Pudientes & $\begin{array}{l}\text { Adinerados, ricos, } \\
\text { acaudalados, opulencia }\end{array}$ & $11.5 \%$ & Trabajadores & Dedicados & $8.3 \%$ \\
\hline Tranquilos & Pasivos, serenos, calmados & $10.6 \%$ & Tranquilos & Pasivos, serenos, calmados & $7.6 \%$ \\
\hline Sociables & Amigables, abiertos, tratables & $8.4 \%$ & Sencillos & Espontáneos & $5.3 \%$ \\
\hline Especiales $^{8}$ & & $5.5 \%$ & Unidos & Compañerismo & $4.3 \%$ \\
\hline Divertidos & Contentos, alegres, joviales & $4.5 \%$ & Groseros & & $3.2 \%$ \\
\hline Cultura & & $3.3 \%$ & Pobres & & $2.9 \%$ \\
\hline Bondadosos & (Buenos, buenas personas) & $3.3 \%$ & Delincuentes & Ladrones, peligrosos, antisociales & $2.8 \%$ \\
\hline Respetuosos & & $3.1 \%$ & Conflictivos & Peleones, problemáticos, relajosos & $2.7 \%$ \\
\hline Desconfiados & & $2.4 \%$ & Fiesteros & Farreros & $2.7 \%$ \\
\hline Egoístas & & $2.4 \%$ & Respetuosos & & $2.7 \%$ \\
\hline Trabajadores & Dedicados & $2.1 \%$ & Solidarios & & $2.7 \%$ \\
\hline Elegantes & Lujosos, sofisticados & $1.8 \%$ & Bondadosos & Buenos, buenas personas & $2.6 \%$ \\
\hline Odiosos & & $1.6 \%$ & Falta de educación & Mal educados & $2.4 \%$ \\
\hline Indiferentes & Apáticos & $1.5 \%$ & Buena onda & Chéveres, bacanes & $2.3 \%$ \\
\hline
\end{tabular}

Fuente: elaboración propia

Cuadro 4.

Matriz comparativa de las características identificadas para la población el "norte" y el "sur"

Términos compartidos

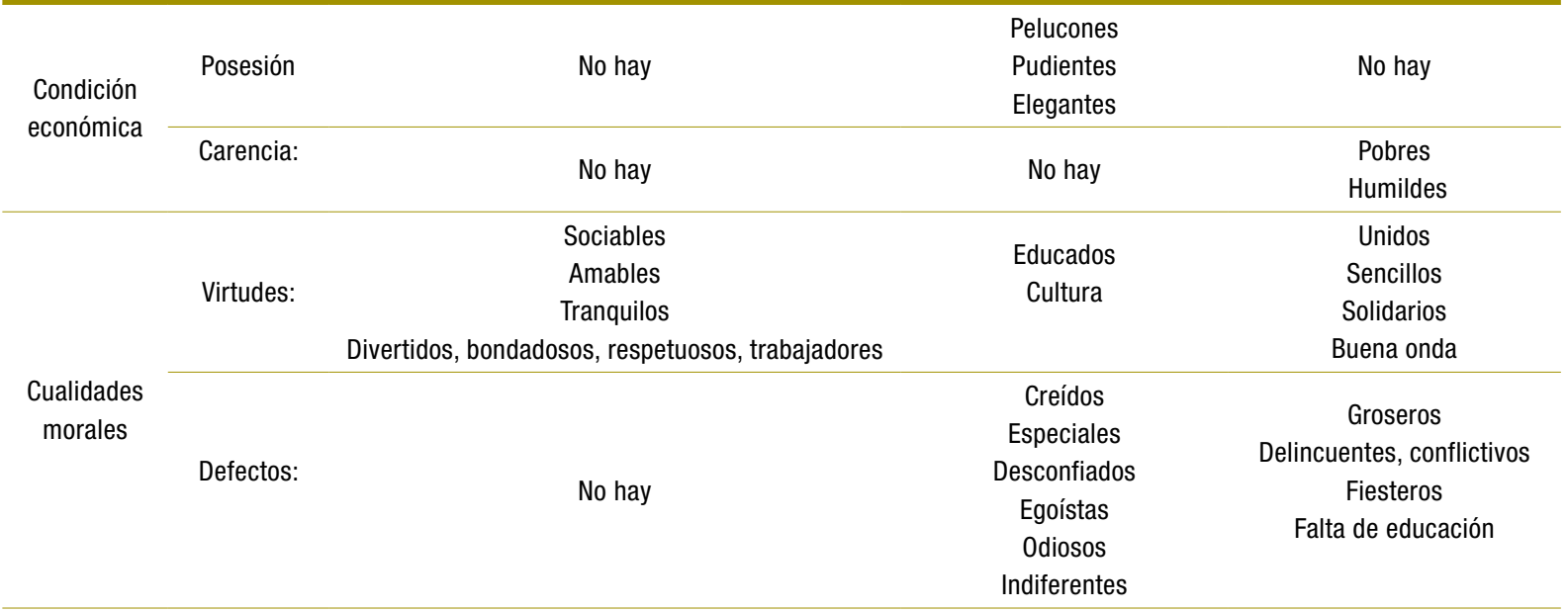


percepción diferenciada de los atributos de la población de ambas zonas se evidencia cuando aparecen tanto virtudes como defectos morales particulares que contribuyen a la formación de la idea del norte y la del sur imaginarios. En el plano de las virtudes morales diferenciadas, la población del Norte es descrita con términos relativos a la idea de capital cultural (Bourdieu, 1988) que remarca la acumulación de educación como elemento de distinción social. En contraste, las virtudes morales particulares de la población Sur remiten a un sentido comunitario de vida asociado con los valores de la cultura popular (Cuadro 4).

El campo de los defectos morales constituye el mayor escenario de disputas de significaciones, pues en él se condensa la mayor cantidad de términos, más allá de su frecuencia estadística. La población del Norte es definida con adjetivos que expresan cierta corrosión de valores morales asociados también con la posesión económica, mientras la población del Sur es referida con defectos vinculados también a la asociación con la carencia de distintos capitales, sean económicos o culturales. Esto muestra otra faceta de las producciones imaginarias en las cuales la significación de la posesión o no de riqueza se traduce al terreno de la moralidad y es ahí donde se logra captar mejor el escenario en disputa: el emplazamiento de las clases sociales en la ciudad de Quito.

Resulta interesante contrastar la forma en la que cada zona se representa a sí misma y como es representada por las otras zonas. Para esto, el análisis hermenéutico antes propuesto se complementa muy bien con el análisis estadístico de la desagregación de las frecuencias por zonas. En este modelo, la caracterización general de población del Norte y las diferencias en su apreciación en los distintos sectores se muestran en el Gráfico 6.
Gráfico 6.

Características de la población del norte

Consolidado de características de la población del norte

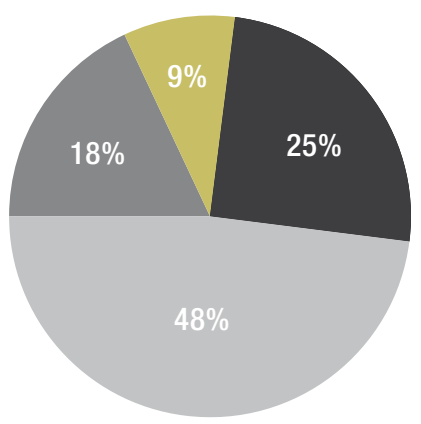

Desagregación por sectores

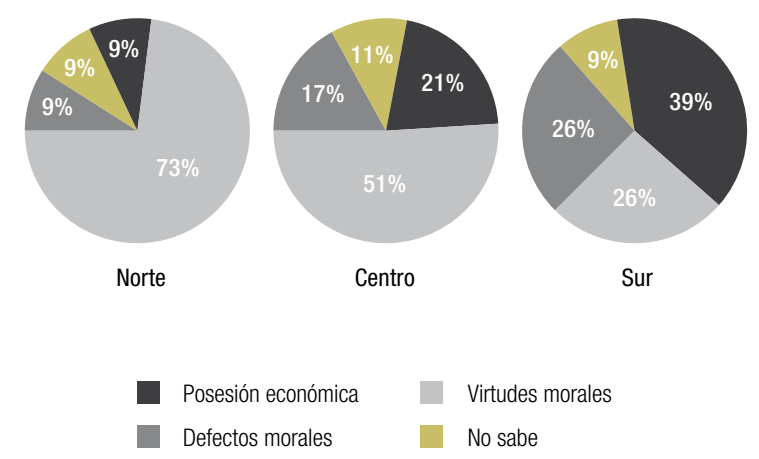

Fuente: Elaboración propia

La autorrepresentación de la población del Norte minimiza los rasgos que aluden a los campos de la posesión económica y de los defectos morales, a la vez que exalta el campo de las virtudes morales; para los otros sectores, estos campos adquieren mayor presencia, sobre todo en la población del Sur. Se observa una polarización entre el modo como la gente del Norte se mira a sí misma y como es vista por la población del Sur, quienes señalan la posesión económica como cualidad de las personas, lo que refuerza la lógica de superponer la caracterización del lugar con la de la población que lo habita. Además, adquiere sentido la crítica implícita en los adjetivos que sancionan la posesión económica, pues el realce 
de los defectos morales deja entrever cierta corrupción moral ligada a la riqueza, expresada en el egoísmo y sentido de superioridad atribuido a las personas del Norte.

El mismo ejercicio de combinar el análisis hermenéutico y estadístico aplicado a la caracterización de la población del Sur se refleja en el Gráfico 7.

Gráfico 7.

Características de las personas del Sur

Consolidado de características de la población del sur

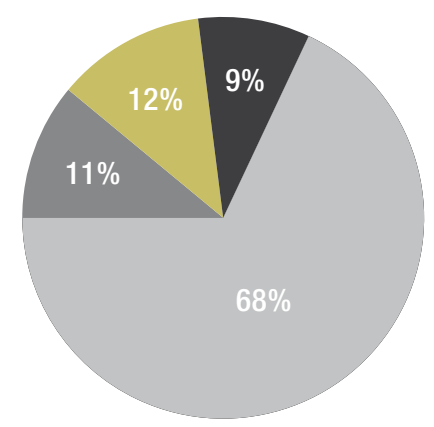

Desagregación por sectores

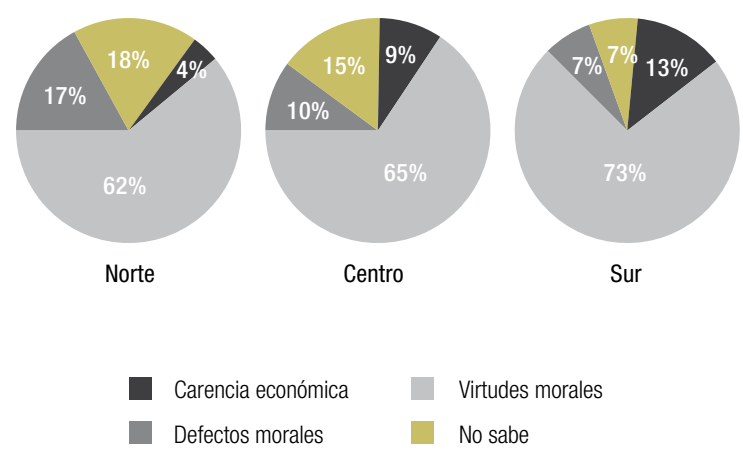

Fuente: Elaboración propia

Acerca del perfil general, las virtudes morales son reconocidas como rasgo de la población del Sur en un rango bastante mayor que en el caso de la caracterización del "norte" y esta apreciación es compartida en los tres sectores. Por su parte, si bien en el análisis hermenéutico se pudo instituir las diferencias cualitativas de los defectos morales respecto a aquellos reconocidos para la población del Norte, en el plano estadístico se observan frecuencias menores en comparación con el "norte". Otro contraste fundamental está en el campo de la condición económica: por un lado está la asociación de la población del Norte con la posesión económica frente a la asociación de la población del Sur con la carencia económica. En el plano estadístico, se aprecia una diferencia importante en la referencia a estos atributos para caracterizar a cada zona, pues, en el caso del "sur", esta referencia resulta minoritaria, a diferencia del "norte", que constituye uno de sus principales atributos.

En la desagregación por sector se puede ver que las diferencias entre zonas no son tan marcadas como en la desagregación de los datos respecto a la población del Norte. En el caso de la primacía de las virtudes morales, la autorrepresentación de la población del Sur enfatiza estos atributos en mayor proporción que la población del Centro y del Norte, al igual que sucede en la autorrepresentación de la población del Norte. El mayor contraste está en que la autorrepresentación de la población del Sur coincide en buena medida con la manera en la que es vista por el resto de la población de la ciudad, lo que implica un importante consenso social; la autorrepresentación de la población del Norte es muy distinta a como es percibida por la población de las otras zonas, lo que da cuenta de que es un campo en disputa.

Otras diferencias importantes en la desagregación son la alusión a la carencia económica como característica de la población del Sur y la incidencia de la respuesta "no sabe", ya que, si bien en ambos casos se trata de frecuencias bajas, las variaciones de las respuestas por sectores son notorias. En el primero, la carencia económica es señalada más por los mismos habitantes del Sur 
que por los de los otros sectores; esto, a su vez, está asociado con la importancia que le da la mirada de la gente del Sur a la posesión económica como cualidad de la población del Norte, lo que implica que, desde su óptica, la condición económica, en general, aparece de manera más resaltada.

En el caso de la respuesta "no sabe" para definir a la población del Sur, se puede observar que varía significativamente entre los sectores, en donde destaca su incidencia en la población del Norte. En contraste, esta opción de respuesta es homogénea en los tres sectores cuando se refiere a la población del Norte.

Si se complementa este dato con la incidencia de la respuesta "no sabe" para caracterizar ambos sectores expuesta en los Cuadros 1 y 2 , se aprecia que está más presente para definir al sur que al norte. Esto quiere decir que la percepción de desconocimiento que fundamenta esta respuesta también está atravesada por la ubicación espacial de las personas encuestadas, en donde la zona Sur y la población que la habita son en mayor medida, desconocidos para la población del Norte, pero no sucede lo mismo en sentido contrario, porque para definir al Norte y a su gente la población del Sur recurre menos a esta expresión.

\section{Conclusiones}

Desde el inicio del diseño de la investigación, se partió de la premisa de que la adscripción territorial incide en la valoración diferenciada entre las zonas norte y sur de la ciudad de Quito, es decir, frente al tema de segregación, las significaciones sobre cada zona están cruzadas en su totalidad por la experiencia urbana, de la que forma parte el lugar de residencia. La desagregación de datos mostrada pone en evidencia que la ubicación del lugar de residencia del encuestado opera como una variable decisiva en la apreciación sobre las diferencias entre cada zona, lo que permite sostener que la producción de significaciones en torno a las divisiones de la ciudad se hace también desde un lugar específico.

Así, es posible hablar de cómo es el norte y el sur para quienes habitan las zonas Norte, Centro y Sur de la ciudad, en donde, si bien hay percepciones hegemónicas que se vuelven transversales en todos los sectores y operan como consensos sociales implícitos, también hay apreciaciones diferenciadas que ponen en disputa las distintas visiones, según desde donde se miran las diferencias socioespaciales.

Lo más destacado es que los datos obtenidos muestran que la población del Norte construye una representación particular de "su" norte distinta a la configurada por la población del Centro y del Sur, en donde sobresale una valoración muy positiva del lugar, pero, en contraste, en la de sus habitantes se expresa una fuerte crítica social a la posesión económica, por medio del señalamiento de múltiples defectos morales asociados con actitudes que demuestran un sentido de superioridad frente al resto de la población.

En el sur, la situación tiende a funcionar en el sentido contrario, pues el campo en disputa es la valoración del lugar antes que la valoración de las personas. En cuanto a la imagen del lugar, en muchos casos los habitantes del sector Sur coinciden en una gran cantidad de aspectos con la visión negativa que tienen de esta zona los residentes del Centro y del Norte, aunque en otros las valoraciones de mayor peso estigmatizante sobre el sur son remarcadas en las percepciones de la población del Norte. Sin embargo, en la valoración de la población del Sur prima una representación bastante positiva, común en los tres sectores, cuya particularidad es que está ligada a "lo popular" como origen de virtudes morales como el comunitarismo, la reciprocidad, la solidaridad, etc., que no solo se consideran perdidas 
en la población del Norte, sino sustituidas por antivalores como el egoísmo, el individualismo y la ostentación de riqueza.

En definitiva, los datos expuestos muestran que, si bien en la actualidad perdura el imaginario dual de Quito como forma de representar las diferencias sociales "espacializadas", su campo de acción no se reduce a la polarización entre el norte rico y el sur pobre, como señalan los estudios previos sobre el caso, sino que la significación de la condición económica está cruzada por valoraciones morales. En este juego, ambas zonas se vuelven ambivalentes: el norte figurativo es imaginado como poseedor de buenas condiciones materiales, pero habitado por personas de escasa sociabilidad y con una especie de complejo de superioridad; en cambio, el sur es visto como desfavorecido, en términos materiales, pero habitado por una población virtuosa en cualidades morales.

Se puede afirmar, de manera categórica, que la ciudad de Quito está atravesada por una división imaginaria, en donde están en juego las características y las valoraciones de ambas zonas; lo que se vuelve irrefutable en el campo de la representación es que sean diferentes y antagónicas. En este escenario, la disputa por la significación termina reforzando la institución de la frontera, porque lo que se vuelve inadmisible para la población de Quito es que ambas zonas sean indiferenciadas. De esta manera, lo más relevante de este antagonismo es que se asienta sobre una matriz binaria común, lo que implica una misma lógica compartida de representación. Como señala Silva (2013): “[...] el ver está reglamentado socialmente, $[\ldots]$ no vemos con los ojos propiamente, $[\ldots]$ los imaginarios nutren las visiones, y por ello las operaciones visuales y cognitivas de la ciudad operan bajo formas recónditas de censura que afectan la percepción" (p. 39).
Desde el marco analítico propuesto, los imaginarios existen como necesidad social de dar sentido a situaciones latentes de conflicto; en esta dirección, el imaginario que tiende a amplificar las diferencias entre ambas zonas y a minimizar sus posibles semejanzas puede interpretarse como un mecanismo para dar forma legible a las diferencias y desigualdades de la población, mediante el uso de las heredadas coordenadas espaciales de norte y sur. No se trata de una representación anacrónica que mantiene una imagen dual de una ciudad que materialmente no se ajusta a esta dicotomía, sino que esta forma de representación responde a una formulación imaginaria que canaliza diversas necesidades de diferenciación social que tienen en común la aversión a construir un sentido de habitar un mismo espacio. En definitiva, el imaginario dual revela que la posibilidad de mixtura social se mantiene como un "fantasma urbano", que marca el sentido de coexistencia de clases en la ciudad de Quito al imaginar espacios diferenciados para cada una.

\section{Bibliografía}

Achig Subía, L. (1983). El proceso urbano de Quito. Un ensayo de interpretación. Quito: Centro de Investigaciones Ciudad.

Aguirre, M., Carrión Mena, F. y Kingman Garcés, E. (2005). Quito imaginado. Bogotá: Universidad Nacional de Colombia, Convenio Andrés Bello, Taurus.

Bourdieu, P. (1988). La distinción: criterios y bases sociales del gusto. Madrid: Taurus.

Carrión Mena, F. (1987). Quito, crisis y política urbana. Quito: El Conejo.

De Mattos, C. (2010). Globalización y metamorfosis urbana en América Latina. Quito: Olacchi, MDMQ. 
Giménez, G. (2001). Cultura, territorio y migraciones. Aproximaciones teóricas. Alteridades, 11(22), 5-14.

Girola, L. (2012). Representaciones e imaginarios sociales. Tendencias recientes en la investigación. En G. de la Garza y E. Leyva (eds.), Tratado de metodología de las Ciencias Sociales: perspectivas actuales (pp. 441-467). México D. F.: FCEUAM Iztapalapa.

Gorelik, A. (2002). Imaginarios urbanos e imaginación urbana: Para un recorrido por los lugares comunes de los estudios culturales urbanos. Eure, XXVIII(83). Recuperado de http://www. redalyc.org/articulo.oa?id=19608308

Hiernaux, D. (2007). Los imaginarios urbanos: de la teoría y los aterrizajes en los estudios urbanos. Eure, XXXIII(99), 17-30.

Ibarra Crespo, H. (1998). La otra cultura. Imaginarios, mestizaje y modernización. Quito: Marka; Abya-Yala.

Kingman Garcés, E. (2006). La ciudady los otros. Quito 1860-1940: higienismo, ornato y policía. Quito: Flacso, Universitat Rovira i Virgili.

Lindón, A. (2012). La concurrencia de lo espacial y lo social. En G. de la Garza y E. Leyva (eds.), Tratado de metodología de las Ciencias Sociales: perspectivas actuales (pp. 584-621). México D. F.: FCE-UAM Iztapalapa.

Naranjo, M. (1999). Segregación espacial y espacio simbólico: un estudio de caso en Quito. En T. Salman y E. Kingman (eds.), Antigua modernidad y memoria del presente: culturas urbanas e identidad (pp. 227-235). Quito: Flacso.

Peña, L. (2011). Algunos elementos metodológicos para pensar espacialmente en Ciencias Sociales. En Centro de Investigaciones sobre Dinámica Social (editor de la serie), Serie II: Vol. 5. Bogotá: Universidad Externado de Colombia, CIDS.

Silva, A. (2004). Imaginarios urbanos: hacia el desarrollo de un urbanismo desde los ciudadanos. Metodología. Bogotá: Convenio Andrés Bello; Universidad Nacional de Colombia.

Silva, A. (2007). Imaginarios urbanos en América Latina: urbanismos ciudadanos. Barcelona: Fundació Antoni Tàpies.

Silva, A. (2013). Imaginarios, el asombro social. Bogotá: Universidad Externado de Colombia.

Unda, M. (1992). Quito, o las dos caras de Dios. Ciudad alternativa, 8(1), 19-32. 


\section{Anexo}

Anexo 1.

Distribución de la muestra aplicada

\begin{tabular}{|c|c|c|c|}
\hline & $\begin{array}{c}\text { Número } \\
\text { de encuestas }\end{array}$ & $\begin{array}{c}(\text { Error mínimo }) \\
(\mathrm{p}=0.05 ; \mathrm{q}=0.95)\end{array}$ & $\begin{array}{l}\text { (Error máximo) } \\
\quad(p=q=0.5)\end{array}$ \\
\hline TOTAL & 930 & +-1.5 & +-3.3 \\
\hline \multicolumn{4}{|l|}{ POR ÁREA URBANA } \\
\hline Sur & 317 & +-2.5 & +-5.8 \\
\hline Centro & 313 & +-2.5 & +-5.8 \\
\hline Norte & 300 & +-2.5 & +-5.8 \\
\hline \multicolumn{4}{|l|}{ POR EDAD } \\
\hline 15 a 29 años & 325 & +-2.5 & +-5.8 \\
\hline 30 a 44 años & 304 & +-2.5 & +-5.8 \\
\hline 45 a 60 años & 301 & +-2.5 & +-5.8 \\
\hline \multicolumn{4}{|l|}{ POR NSE } \\
\hline Alto & 160 & +-3.6 & +-8.2 \\
\hline Medio & 363 & +-2.3 & +-5.3 \\
\hline Bajo & 407 & +-2.2 & +-5.0 \\
\hline \multicolumn{4}{|l|}{ POR SEXO } \\
\hline Hombres & 466 & +-2.1 & +-4.7 \\
\hline Mujeres & 464 & +-2.1 & +-4.7 \\
\hline
\end{tabular}

